\title{
Enhanced hard-probe measurements in the 2020s with the ALICE Upgrade
}

\author{
Cristina Bedda (for the ALICE Collaboration)* \\ Utrecht University \\ E-mail: cristina.bedda@cern.ch
}

The ALICE Collaboration is preparing a major upgrade of the detector apparatus during the second LHC long shutdown (LS2, 2019-20) in view of the LHC Runs 3 and 4 (2021 to 2029).

The most relevant aspects of this upgrade program are described in this contribution, together with some selected items from the physics program.

International Conference on Hard and Electromagnetic Probes of High-Energy Nuclear Collisions 30 September - 5 October 2018

Aix-Les-Bains, Savoie, France

${ }^{*}$ Speaker. 


\section{Introduction}

The ALICE Collaboration is preparing a major upgrade of the detector apparatus during the second LHC long shutdown (LS2, 2019-20) [1] in view of the LHC Runs 3 and 4 (2021 to 2029).

The objective of the ALICE upgrade for LS2 is two-fold: i) an improvement of the tracking precision and efficiency, in particular in the low-momentum range; ii) an improvement of the readout capabilities of the experiment, to fully exploit the LHC luminosity for heavy ions envisaged after LS2.

The first goal will be achieved by replacing the Inner Tracking System with a new tracker, composed of seven cylindrical layers of monolithic silicon pixel detectors (MAPS) with fast readout, high granularity and low material thickness [2], and by introducing a new telescope tracker, also composed of MAPS, in front of the muon spectrometer [3]. As an example, the resolution of the track spatial position will improve by about a factor of three in the direction transverse to beam line and a factor of five in the longitudinal direction. The second goal will be achieved by replacing the readout chambers of the Time Projection Chamber with Gas Electron Multiplier (GEM) detectors [4], by upgrading or replacing the readout electronics of several of the other detector systems, by adding a new fast trigger detector [5] and by implementing a new integrated online-offline architecture [6]. The upgraded ALICE detector will be capable of reading out $\mathrm{Pb}-\mathrm{Pb}$ interactions in minimum-bias trigger mode at a rate of $50 \mathrm{kHz}$, corresponding to 100 times the current rate.

The prime physics goals of the ALICE upgrade are high-precision measurements of heavyflavour, charmonium and low-mass di-lepton production, with particular emphasis on the low transverse momentum $\left(p_{\mathrm{T}}\right)$ region. In the following some selected items from the foreseen physics program are shown in both $\mathrm{Pb}-\mathrm{Pb}$ and $\mathrm{p}-\mathrm{Pb}$ collisions. Additional material on the projections for hadron-jet correlations can be found in [7].

\section{Heavy flavour production}

A strong improvement in the heavy-flavour sector is expected after the ALICE Upgrade. As an example the expected performance with integrated luminosity of $L_{\mathrm{int}}=10 \mathrm{nb}^{-1}$ for the nuclear modification factor $\left(R_{\mathrm{AA}}\right)$ and the elliptic flow $\left(v_{2}\right)$ of charmed and beauty hadrons are shown in Fig. 1. It will be possible to measure the $R_{\mathrm{AA}}$ of $\mathrm{D}^{0}$ down to $p_{\mathrm{T}}=0$ in $\mathrm{Pb}-\mathrm{Pb}$ collisions, obtaining an extended and more precise measurement with respect to the current results. In addition, ALICE
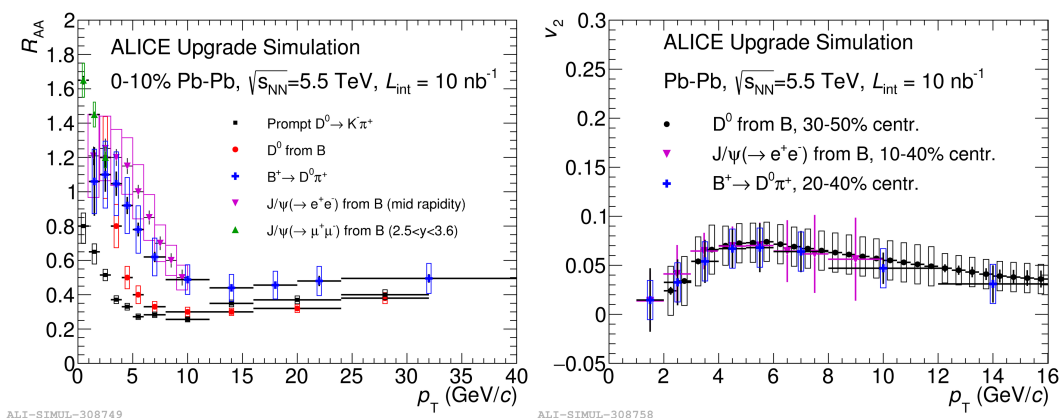

Figure 1: Expected performance for the measurement of the $R_{\mathrm{AA}}$ and $v_{2}$ of charmed and beauty hadrons. 

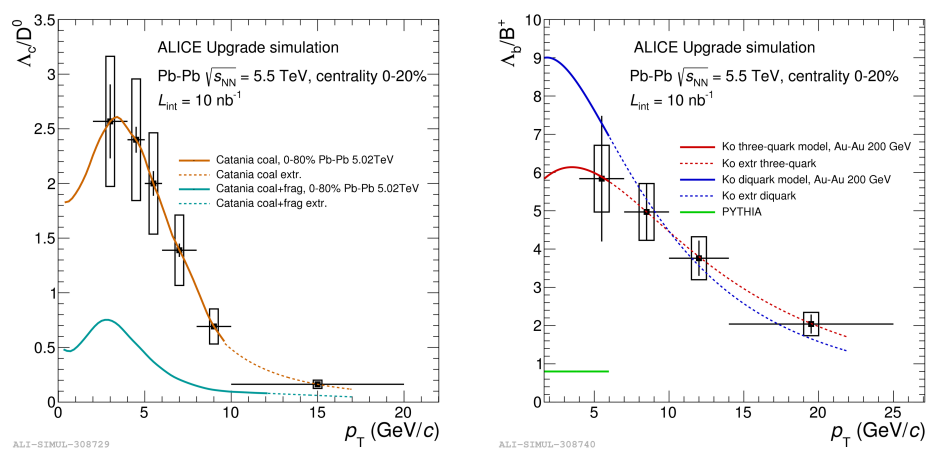

Figure 2: Expected performance for the measurements of the heavy flavour baryon-to-meson ratio: $\Lambda_{\mathrm{c}}^{+} / \mathrm{D}^{0}$ ratio superimposed with the Catania model [9] including coalescence and/or fragmentation processes (left), and $\Lambda_{\mathrm{b}}^{+} / \mathrm{B}^{+}$ratio superimposed with PYTHIA calculations and different implementations of the model described in [10] (right).

plans to measure beauty production with different approaches: inclusive decays, as $\mathrm{B}^{+} \rightarrow \mathrm{D}^{0} \pi^{+}$, and exclusive decays, as non-prompt $\mathrm{D}^{0}$ and non-prompt $\mathrm{J} / \psi$ at $\operatorname{mid}\left(\mathrm{J} / \psi \rightarrow e^{+} e^{-}\right)$and forward $\left(\mathrm{J} / \psi \rightarrow \mu^{+} \mu^{-}\right)$rapidity. The level of precision with the LHC Run 3 and 4 data will allow to quantify the mass dependence of energy loss and to discriminate between energy loss models. In addition, the $v_{2}$ measurements down to 0 and $1 \mathrm{GeV} / c$ for charm and beauty hadrons, respectively, will provide the strongest constraint on the $\mathrm{c}$ and $\mathrm{b}$ quark diffusion coefficients and path-length dependence of the parton energy loss. The measurement of the baryon-to-meson ratio in the heavy flavour sector will also be improved. Currently the $\Lambda_{c}^{+} / \mathrm{D}^{0}$ ratio has been measured at RHIC and LHC in heavy-ion collisions and a hint of charm recombination has been observed but the measurements are affected by large statistical uncertainties [8]. After the ALICE Upgrade, it will be possible to measure the $\Lambda_{\mathrm{c}}^{+} / \mathrm{D}^{0}$ ratio down to $p_{\mathrm{T}}=2 \mathrm{GeV} / c$, as shown in Fig. 2 (left), and, for the first time in $\mathrm{Pb}-\mathrm{Pb}$ collisions, the $\Lambda_{\mathrm{b}}^{+} / \mathrm{B}^{+}$ratio will also be available, as shown in Fig. 2 (right). Those measurements will allow to discriminate between the models at low $p_{\mathrm{T}}$ and they will provide insight into the thermalisation and hadronisation processes for both charm and beauty quarks.

\section{Quarkonia}
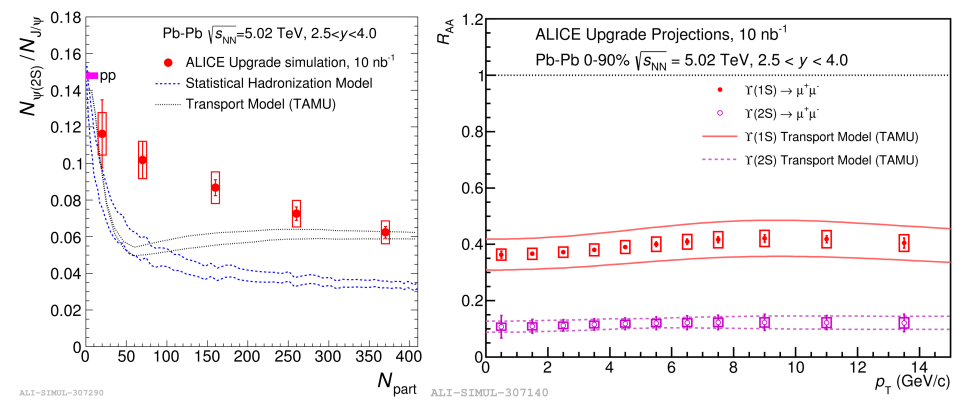

Figure 3: Expected performance of the $\psi(2 \mathrm{~S})$ and $\mathrm{J} / \psi$ yields ratio (left), and of the $R_{\mathrm{AA}}$ of bottomonium states (right). 


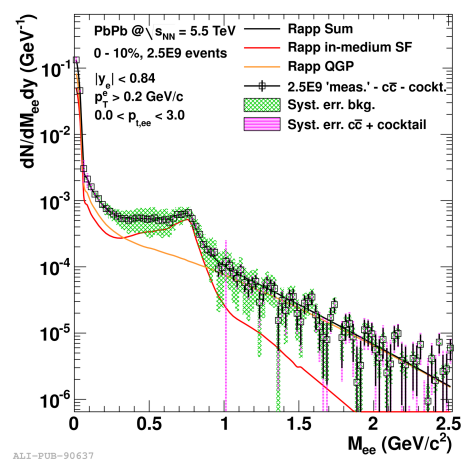

Figure 4: Expected performance for the measurement of the low-mass di-electron spectrum with the upgraded ALICE detector [2].

Measurements of charmonium and bottomonium states allow to probe the modification of the QCD potential in the QGP, which is also sensitive to its temperature, through quarkonium sequential dissociation pattern and regeneration. After the ALICE Upgrade, it will be possible to measure $\psi(2 \mathrm{~S})$ production with small uncertainties, as shown in Fig. 3 (left), allowing the study of the two different scenarios that aim at describing charmonia data: the stastical hadronisation model [11] and the transport model [12]. It will also be possible to measure excited bottomonium states with uncertainties smaller than the ones currently provided by the models. An example is shown in Fig. 3 (right) with the expected performance of the $\Upsilon(1 \mathrm{~S}) R_{\mathrm{AA}}$. In addition, it will be possible to perform more differential measurements, including the elliptic flow of bottomonium states.

\section{Low mass di-leptons}

Low mass di-leptons are a powerful tool to study the properties of the Quark-Gluon-Plasma because they are emitted through the whole evolution of the medium. Their study requires very efficient background reduction and a very large sample of minimum-bias data. Currently, the measurement of the low mass di-leptons in $\mathrm{Pb}-\mathrm{Pb}$ collisions is affected by several electron-background sources and by limited statistics. The expected performance for the measurement of the low-mass di-electron spectrum after the ALICE Upgrade is shown in Fig. 4. Thanks to the new Inner Tracking System, it will be possible to better reject the conversion and charm backgrounds and reduce the combinatorial background. A dedicated run with a lower magnetic field $(B=0.2 \mathrm{~T})$ is foreseen in order to extend the electron $p_{\mathrm{T}}$ range and acceptance.

\section{Measurements in p-Pb collisions}

One of the open questions in high-energy nuclear physics is the understanding of the degree of thermalisation and collective-like behaviour in small system. During the LHC Run 3 and 4 ALICE will collect a data sample with an integrated luminosity of $L_{\text {int }}=500 \mathrm{nb}^{-1}$ of minimum-bias $\mathrm{p}-\mathrm{Pb}$ collisions with the purpose to investigate initial and final state effects in different sectors. The expected performance for the measurement of the D-meson $R_{\mathrm{pPb}}$ compared with the current preliminary results is shown in Fig. 5 (left), the precision achievable will allow to disentangle 

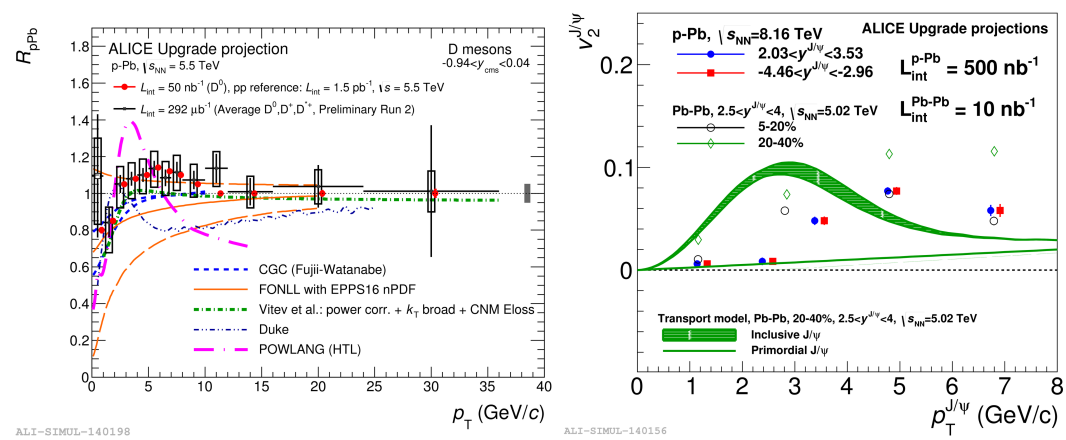

Figure 5: Expected performance for the measurement of the $p_{\mathrm{T}}$ dependence of the D-meson $R_{\mathrm{pPb}}$ compared with the current preliminary result [13] (left), and of the $\mathrm{J} / \psi v_{2}$ in different rapidity ranges compared to the expectation in $\mathrm{Pb}-\mathrm{Pb}$ collisions (right).

between models including cold-nuclear-matter effects and/or the formation of a small region of Quark-Gluon-Plasma. In addition, more differential measurements will be possible to investigate initial/final state effects in high-multiplicity $\mathrm{p}-\mathrm{Pb}$ collisions. Collective-like effects in $\mathrm{p}-\mathrm{Pb}$ collisions will also be investigated through the measurements of the elliptic flow of open and hidden heavy-flavour hadrons. As an example, the expected performance for the $\mathrm{J} / \psi v_{2}$ measurement is shown in Fig. 5 (right).

\section{Conclusions}

The ALICE Upgrade project is well on track for installation during the second LHC long shutdown. The upgraded detectors and the higher statistics of the LHC Runs 3 and 4 will allow to perform high precision measurements of heavy-flavours, quarkonia, jets and low-mass dileptons observables.

\section{References}

[1] ALICE Collaboration, CERN-LHCC-2012-012.

[2] ALICE Collaboration, J. Phys. G: Nucl. Part. Phys. 41 (2014) 087002.

[3] ALICE Collaboration, CERN-LHCC-2015-001.

[4] ALICE Collaboration, CERN-LHCC-2013-020.

[5] ALICE Collaboration, CERN-LHCC-2013-019.

[6] ALICE Collaboration, CERN-LHCC-2015-006.

[7] J. Norman, These proceedings, (2018).

[8] ALICE Collaboration, arXiv:1809.10922.

[9] K. Das et al., PRD 90 (2014) 054018.

[10] Y. Oh et al., PRC 79 (2009) 044905.

[11] A. Andronic et al., Nucl. Phys. A904-905 (2013) 535c-538c.

[12] X. Zhao and R. Rapp, Nucl. Phys. A859 (2011) 114-125.

[13] ALICE Collaboration, ALICE-PUBLIC-2017-008. 